\title{
Modeling polycrystalline materials with elongated grains
}

\author{
Irvin Pérez ${ }^{1}$ | Márcio Muniz de Farias ${ }^{2,3} \mid$ Manuel Castro $^{1}$ | Roberto Roselló ${ }^{1}$ | \\ Carlos Recarey Morfa ${ }^{1,3}$ | Liosber Medina ${ }^{4}$ | E. Oñate ${ }^{5}$
}

${ }^{1}$ Center of Computational Mechanics and Numerical Methods in Engineering, CIMNE-UCLV Classroom, Central University of Las Villas, Santa Clara, Cuba

${ }^{2}$ InfraLab, University of Brasilia, Brasilia, Brazil

${ }^{3}$ Faculty of Technology, University of Brasilia, Brasilia, Brazil

${ }^{4}$ Department of Transport Engineering, Federal University of Goiás, Goiânia, Brazil

${ }^{5}$ International Center for Numerical Methods in Engineering, Polytechnic University of Catalonia, Barcelona, Spain

\section{Correspondence}

Irvin Pérez, Center of Computational Mechanics and Numerical Methods in Engineering, CIMNE-UCLV Classroom, Central University of Las Villas,

Santa Clara, Cuba.

Email: hotei.mat@gmail.com

\section{Funding information}

Coordination for the Improvement of Higher Education Personnel (CAPES), Brazilian Ministry of Education, Grant/Award Number: CAPES-MES Project No. 208/13; InfraLab, Faculty of Technology, University of Brasilia; International Center for Numerical Methods in Engineering (CIMNE)

\section{1 | INTRODUCTION}

The behavior of the microstructure of some polycrystalline materials can be successfully simulated with the finite element method, ${ }^{1-3}$ by modeling a representative volume element (RVE) ${ }^{4}$ For this purpose, the medium should be represented by partitioning the domain into polyhedra. One way to obtain such partition can be by obtaining polyhedra that are circumscribed to bodies of a packing that fills the domain with a high volume fraction (see Figure 2 bottom for an example in 2D). The sizes and shapes of the polyhedra must follow some given statistical distribution, and they are determined by the elements of the packing contained inside the polyhedra.

Spheres are one of the simplest shapes for generating a dense packing ${ }^{5}$ and obtaining a polyhedral partition from it. Once a sphere packing exists, there are two main approaches for calculating the polyhedral partition from it. The first approach $^{1}$ consists in calculating a power diagram (also known as Laguerre diagram), which can be obtained by relocating 
the cell vertices of the Voronoi tessellation of the spheres' centers. It has the disadvantage that, in 3D, it is only suitable for spheres. The second approach ${ }^{6}$ is more general and consists in iteratively calculating the planes defining the faces of each cell. This method is the one implemented in the popular library Voro $++^{7}$ for spheres.

Two other publicly available computational libraries for calculating Voronoi tessellations must be mentioned. The first one is CGAL, ${ }^{8}$ which allows the calculation of both weighted diagrams and diagrams with respect to segments. The other one is Qhull, ${ }^{9}$ which allows calculating Voronoi diagrams and also convex hulls. However, none of them produce tessellations in 3D from sets of shapes other than spheres.

When the centers of a Voronoi diagram are sets other than single points, the tessellation may have the disadvantage of containing cells with curved faces. However, the interested reader can check the work of Emiris et $\mathrm{al}^{10}$ for obtaining such diagrams for pseudo-circles and the work of Dong et $\mathrm{al}^{11}$ for arbitrary shapes as well. The former method is based on a polynomial representation of the diagram, while the latter is based on a pixel approximation.

Polycrystalline materials do not always have grains with aspect ratios close to 1 . However, as far as we can tell, no previous results have been presented to model polycrystalline materials with grains having aspect ratios very different from 1, by means of tessellations built from dense packings of noncircular shapes. In this paper, an initial attempt in that direction is presented, by using dense packings of noncircular shapes such as ellipses and spherocylinders in order to obtain the tessellations.

\section{2 | MODELING POLYCRYSTALLINE MATERIALS}

The whole process of modeling granular solids using microscopic images, according to the works of Benabbou et al, ${ }^{1}$ $\mathrm{Hitti}^{2}$ and Morfa et al, ${ }^{3}$ can be summarized in the following Pseudocode 1.

Step 1) Acquisition of microscopic images

Step 2) Grain segmentation

Step 3) Estimation of statistical distribution $D$ of grain sizes

Step 4) Generation of a packing of spheres, as dense as possible, whose radii follow distribution $D$

Step 5) Construction of a Voronoi diagram whose centers are the sphere's centers

Step 6) Construction of a Laguerre diagram obtained by repositioning the vertices of the Voronoi diagram obtained in the previous step, in such a way that each vertex is equidistant from three spheres with respect to the additively weighted power distance.

Pseudocode 1. Virtual geometrical modeling of polycrystalline structures

The first two steps of the previous pseudocode will not be explained in detail since they are out of the scope of the present paper and consist in the acquisition and segmentation of images of polycrystalline materials (Figure 1). This segmentation allows to estimate the grain size distribution (step 3) that can be used as input for a sphere packing algorithm, in order to obtain a dense sphere packing whose sizes have the same distribution as the grains of the microscopical original image (step 4 and Figure 2 top). The packing of spheres allows the calculation of a Laguerre diagram of polyhedral cells containing the spheres (step 6 and Figure 2), as explained in the following.
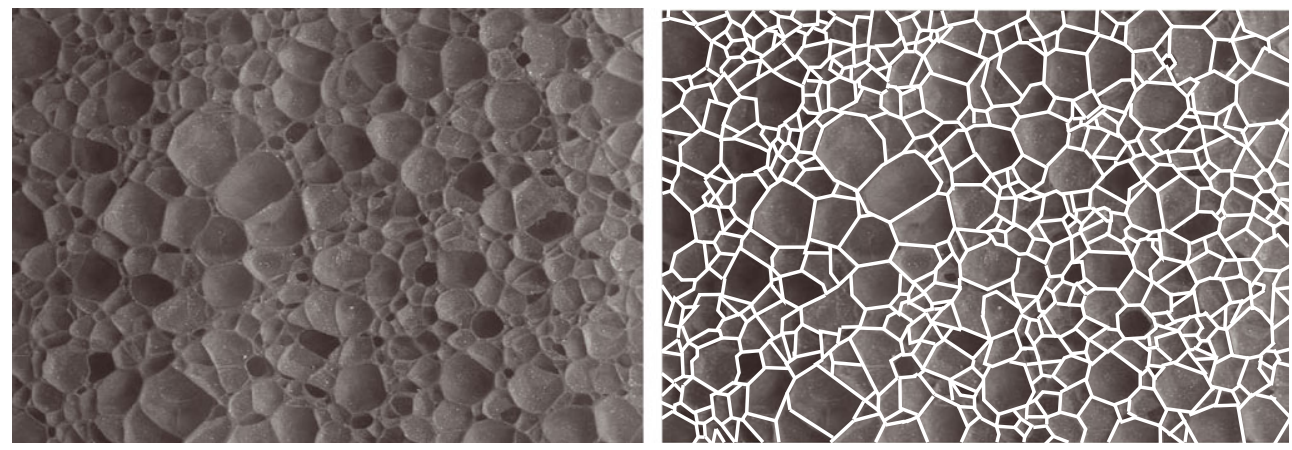

FIGURE 1 Polycrystalline material: closed cell foam. ${ }^{2}$ Left: original 2D image. Right: segmented image 


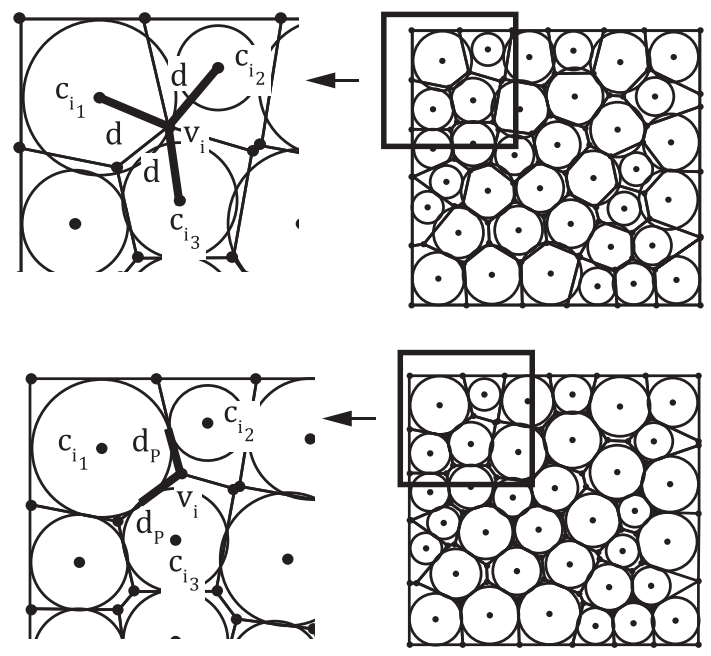

FIGURE 2 Details of Laguerre diagram obtained from a dense set of disks. (A) Packing with Voronoi tessellation superimposed. (B) Packing with Laguerre tessellation superimposed

The algorithm chosen to generate the packings in this research is an advancing front algorithm that allows to generate high density packings of bodies having an arbitrary distribution of shape and size, in reasonable times. ${ }^{5,12,13}$ Such algorithm is a good cost-effective alternative against other methods like gravity deposition, lattice methods, or methods to solve the bin-packing problem.

The additively weighted power distance (AWPD) $d_{P}(\mathbf{p}, \mathbf{c})$ between two points $\mathbf{p}$ and $\mathbf{c}$ is defined by the expression $d_{P}(\mathbf{p}, \mathbf{c})=d(\mathbf{p}, \mathbf{c})^{2}-w^{2}$, where $d$ is the Euclidean distance and $w$ is any positive number. ${ }^{14}$ If $\mathbf{c}$ is the center of a circle $C$ of radius $r$ and $w=r$, then $d_{P}(\mathbf{p}, \mathbf{c})$ is equal to the squared length of the tangent line that goes from $\mathbf{p}$ to $C$, as a consequence of the Pythagoras theorem, and $d_{P}(\mathbf{p}, \mathbf{c})$ can be considered as the AWPD between $\mathbf{p}$ and $C$.

Let $\mathbb{V}$ be a Voronoi diagram whose centers are the centers of a set of circles, like the one of Figure 2 top. Each Voronoi cell has to contain exactly one of the circle's centers, and each vertex of each Voronoi cell must be equidistant from three circle's centers, but the circles do not have to necessarily be contained in the cells, as can be seen in that figure. However, the cells' vertices can be modified for this to happen. Let $\mathbf{v}_{\mathbf{i}}$ be a vertex of $\mathbb{V}$ equidistant with respect to the Euclidean distance $d$ from three circle centers $\mathbf{c}_{\mathbf{i}_{1}}, \mathbf{c}_{\mathbf{i}_{2}}$, and $\mathbf{c}_{\mathbf{i}_{3}}$, being $r_{1}, r_{2}$, and $r_{3}$ the respective radii (Figure 2 top). If each vertex $\mathbf{v}_{\mathbf{i}}$ of $\mathbb{V}$ is transformed into a vertex $\mathbf{v}_{\mathbf{i}}^{\prime}$ equidistant from $\mathbf{c}_{\mathbf{i}_{1}}, \mathbf{c}_{\mathbf{i}_{2}}$, and $\mathbf{c}_{\mathbf{i}_{3}}$ with respect to the AWPD $d_{P}$ with weights equal to $r_{1}$, $r_{2}$, and $r_{3}$, respectively, then the cells of the resulting tessellation (known as Laguerre diagram) will contain the circles, as can be seen in Figure 2 bottom. The 3D case of spheres is totally analogous.

With the previous steps, a virtual reproduction of polycrystalline materials can be obtained. However, this approach has the disadvantage that it will not be realistic for polycrystalline materials having elongated grains, such as the ones in Figure 3. ${ }^{15}$ The figure shows the microstructure of an ultrafine-grained Ni microstructure after strains of $1600 \%$ and $3200 \%$. One solution to this problem is to modify step 4 of Pseudocode 1 by generating packings of nonspherical particles having the same aspect ratio distribution as the polyhedral grains and to modify step 6 of Pseudocode 1 by using a distance other than the AWPD.

\section{3 | MODIFICATION OF THE VORONOI DIAGRAM IN 2D}

Suppose that a Voronoi diagram has been calculated with respect to the centers of a set of particles and that it is necessary to modify the diagram in such a way that its cells fully contain the particles. A possibility to accomplish this could be to modify each Voronoi vertex so as to make it equidistant from the bodies that contain the centers from which it was originally equidistant. Figure 2 shows an example of this modification, where the particles are circles and each relocated Voronoi vertex is equidistant from three circles with respect to the AWPD. ${ }^{1}$ In the top tessellation, each vertex (which is not in the boundary box) is equidistant from three disk centers, and in the bottom tessellation, each vertex is equidistant from three disks with respect to the AWPD and the particles are fully contained in the cells. In this case of circular particles, the AWPD was used to calculate the distance from a point to a body, but for other particle shapes, other distances can be used. 


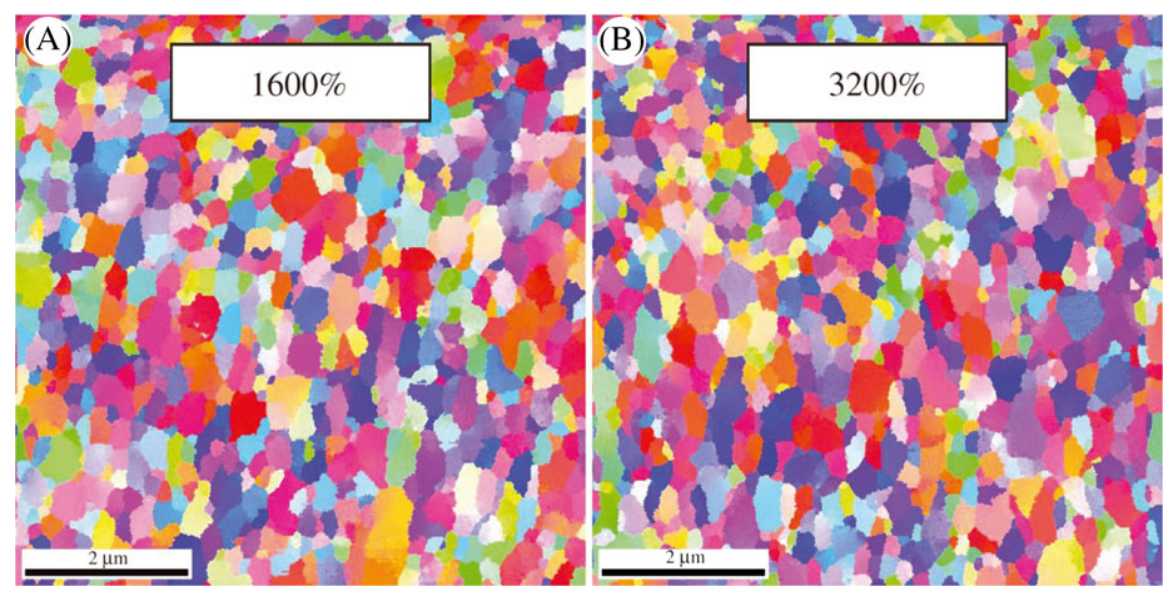

FIGURE 3 Example of an ultrafine-grained Ni microstructure after strains of (A) 1600\% and (B) 3200\% (see the work of Hohenwarter and $\operatorname{Pippan}^{15}$ ) [Colour figure can be viewed at wileyonlinelibrary.com]
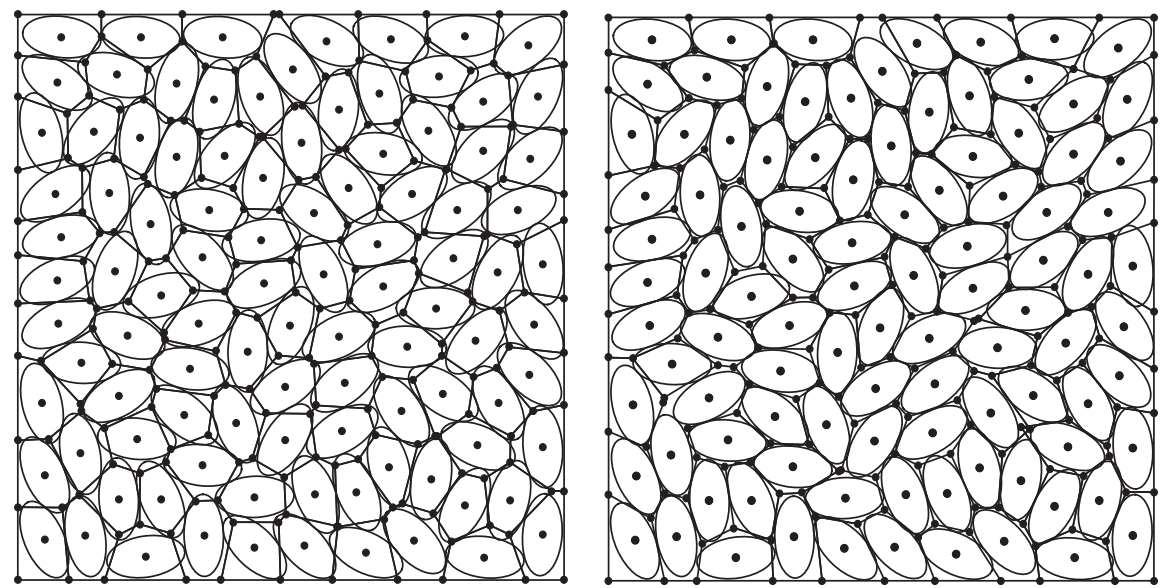

FIGURE 4 Left: Voronoi diagram with respect to the centers of a set of ellipses. Right: tessellation with cells containing the ellipses

A more natural way to define or calculate the distance from a point to a body is the infimum distance from the point to the body. If $\mathbf{p}$ and $B$ are a point and a set of $\mathbb{R}^{n}$, respectively, then the infimum distance $d_{\inf }(\mathbf{p}, B)$ from $\mathbf{p}$ to $B$ is defined by the expression

$$
d_{\mathrm{inf}}(\mathbf{p}, B)=\inf _{\mathbf{q} \in B} d(\mathbf{p}, \mathbf{q}),
$$

where $\mathbf{q}$ is any point (or element) of $B$ and $d$ is the Euclidean distance in $\mathbb{R}^{n}$. The equality $d_{\text {inf }}(\mathbf{p}, B)=d(\mathbf{p}, \mathbf{q})$ holds for some point $\mathbf{q}$ in (the border of) $B$ if $B$ is a closed set. Using this definition, Voronoi diagrams have been readjusted for packings of ellipses and spherocylinders, which are elongated shapes. Examples for these shapes can be seen in Figures 4 and 5, which have the Voronoi diagram to the left and the corresponding modified tessellation to the right.

Ellipses are a very common conic section, while a spherocylinder is a capsule-like body determined by a line segment and a positive real number called radius. It is defined as the set of all points that lie at a distance from the segment equal to or smaller than the radius. Expression (1) for ellipses can be found by minimization. ${ }^{16}$ On the other hand, the distance from a point to a spherocylinder is the distance from the point to the line segment that determines the spherocylinder, minus its radius.

Despite all the 2D examples in this paper are based on the data of Figures 4 and 5, where equal particles have orientations distributed according to the continuous uniform distribution on interval $[0,2 \pi]$, it is no problem at all to base the analysis on different sized particles and other types of orientations, such as in Figure 6. High density packings with arbitrary sizes and orientations can be relatively easily obtained with advancing front packing algorithms such as those in the works of Morales et $\mathrm{al}^{12}$ and Hohenwarter and Pippan. ${ }^{16}$ 

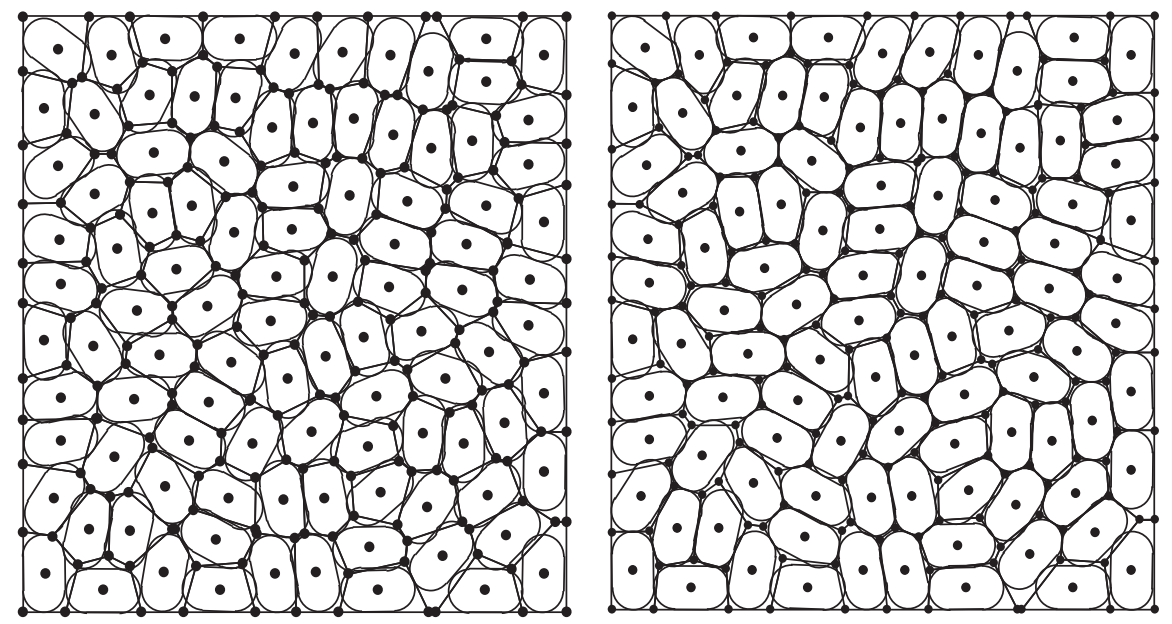

FIGURE 5 Left: Voronoi diagram with respect to the centers of a set of spherocylinders. Right: tessellation with cells containing the spherocylinders

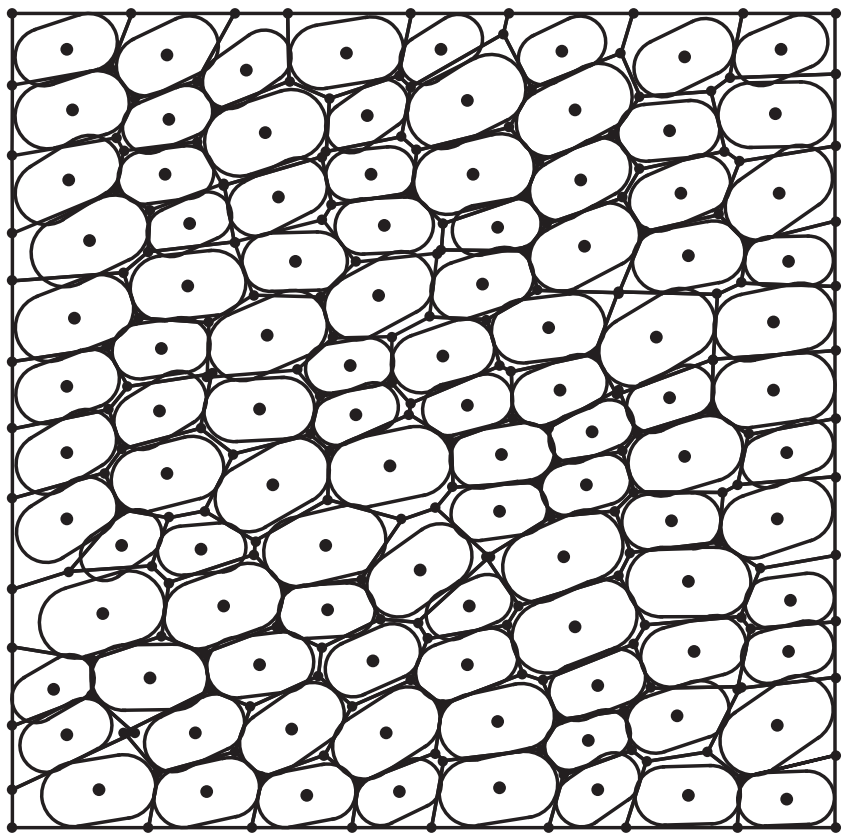

FIGURE 6 Tessellation containing different sized spherocylinders with a fairly common orientation

\section{QUANTIFICATION OF THE RESEMBLANCE BETWEEN THE SHAPES OF A PACKING IN 2D AND THE CELLS OF A TESSELLATION}

It may be useful to evaluate the resemblance between the cells of a tessellation and the particles they contain. This helps choosing the most appropriate body shapes to generate the packing and contributes to make the subsequent physical simulations more realistic. One way to measure the resemblance between two sets $A$ and $B$ can be the Jaccard similarity coefficient, ${ }^{17}$ which is defined by the expression

$$
J(A, B)=\frac{M(A \cap B)}{M(A \cup B)}
$$

where $M(X)$ denotes the area or volume of set $X$ in case $X \in \mathbb{R}^{2}$ or $X \in \mathbb{R}^{3}$, respectively. The components of the $J$ coefficient are illustrated in Figure 7, for an elliptical grain $A$ overlapping a hexagonal cell $B$. Of course, similarity $J$ should be equal to or smaller than one and greater than zero. 

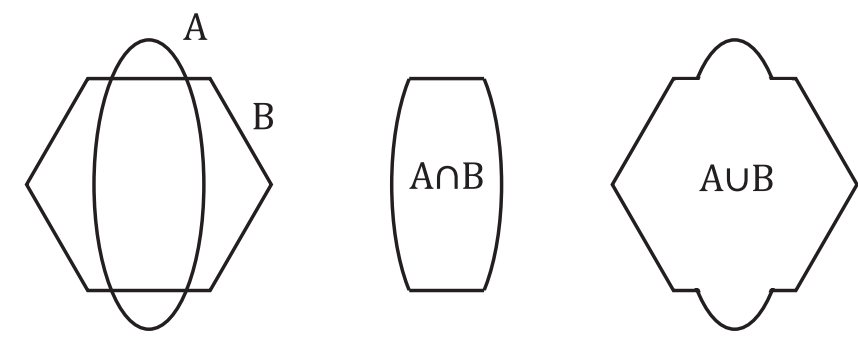

FIGURE 7 Components of the Jaccard coefficient. Two bodies $A$ and $B$ (left); their intersection $A \cap B$ (center) and their union $A \cup B$ (right)

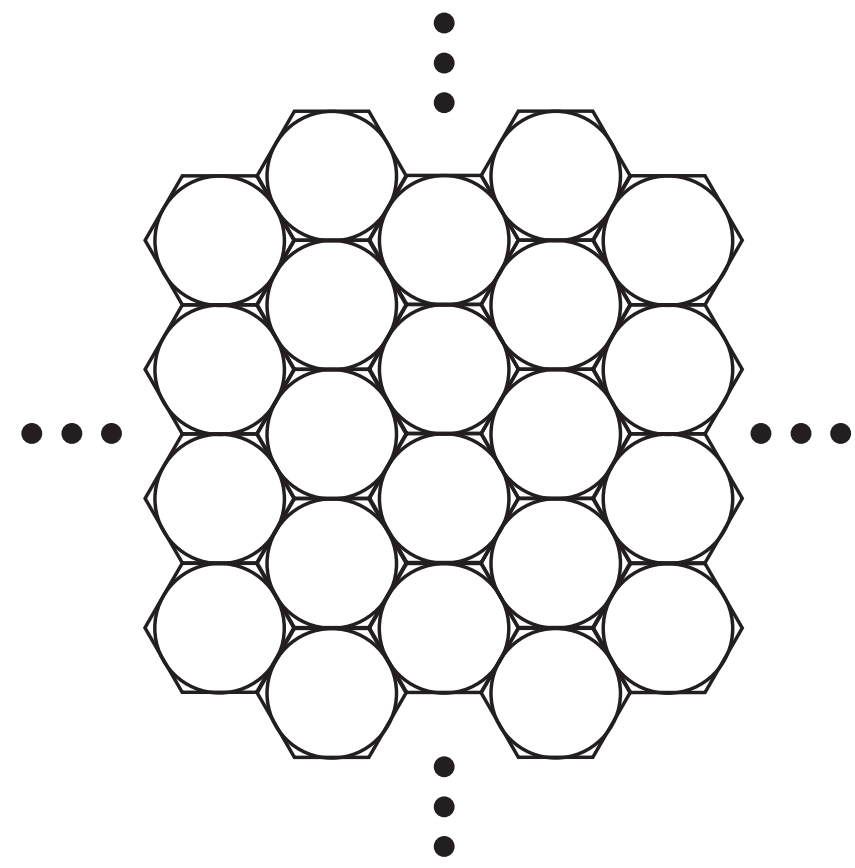

FIGURE 8 Power diagram built from an infinite hexagonal arrangement of disks

A useful benchmark for assessing a tessellation in 2D can be the power diagram built from an infinite honeycomb hexagonal arrangement of equal disks (Figure 8), which has, according to formula (2), a similarity of $\frac{\pi}{2 \sqrt{3}}=0.91$ between each disk and the cell that contains it. Such packing has the highest possible similarity for equal disks in the plane. ${ }^{18}$ In the analogous 3D case, the maximum possible similarity is 0.74 , according to Kepler's conjecture, ${ }^{19}$ being the cells irregular dodecahedra. This maximum similarity value can be achieved with both the cubic close packing ${ }^{20}$ and the hexagonal close packing $^{21}$ (Figure 9 left and right, respectively).

Let $V_{i}, i=\overline{1, n}$, be the Voronoi cells containing the centers of a set of disjoint bodies $B_{i}$, respectively, and let $L_{i}$ be the cell in a tessellation obtained from $V_{i}$ for each $i \in\{1, \ldots, n\}$, respectively, as explained in the previous section. Then, it makes sense to expect in practice the following inequalities to hold:

$$
0<J\left(V_{i}, B_{i}\right)<J\left(L_{i}, B_{i}\right)<J_{\max }=\left\{\begin{array}{ll}
0.91, & \text { if the space dimension is } 2 \\
0.74, & \text { if the space dimension is } 3
\end{array}<1,\right.
$$

which means that the similarity between a cell in the modified Voronoi tessellation and its associated body should usually be greater than the similarity between that same body and the associated Voronoi cell but should usually be smaller than the similarity between the cells and the respective bodies in a hexagonal packing. An illustration of this idea in 2D can be seen in Figure 10, which shows, to the left, a Voronoi cell $V_{i}$ containing the center of an ellipse $B_{i}$, in the center, a cell $L_{i}$ of the tessellation obtained from the Voronoi diagram, containing $B_{i}$, and to the right, a hexagonal cell containing a disk. However, the third inequality from left to right in (3) might not hold in some cases, given that some shapes could be more adjusted to the containing cell than a circle or a sphere in a lattice packing is, and that is why a few spikes in Figures 12, 13 , and 15 are above the thresholds of 0.91 (2D cases) or 0.74 (3D case). 

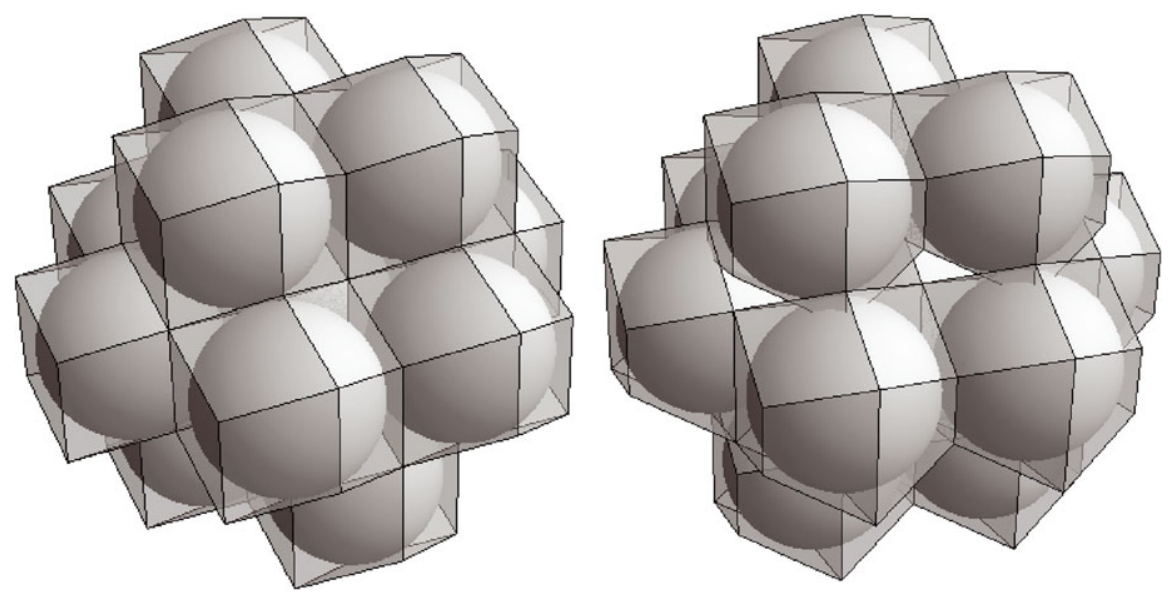

FIGURE 9 Samples of the infinite cubic close packing (left) and hexagonal close packing (right) of spheres, with their respective Voronoi tessellation of irregular dodecahedra cells

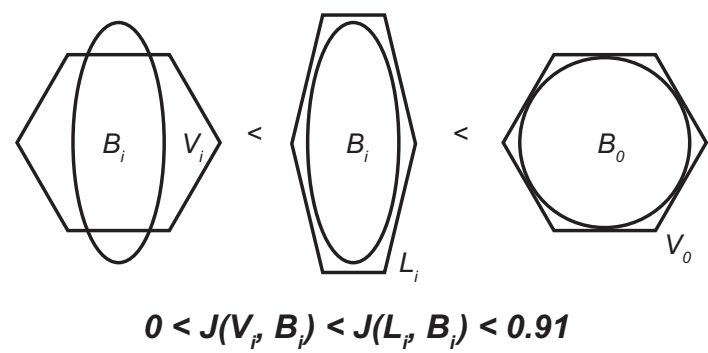

FIGURE 10 Similarity between cells and bodies in Voronoi and power diagrams

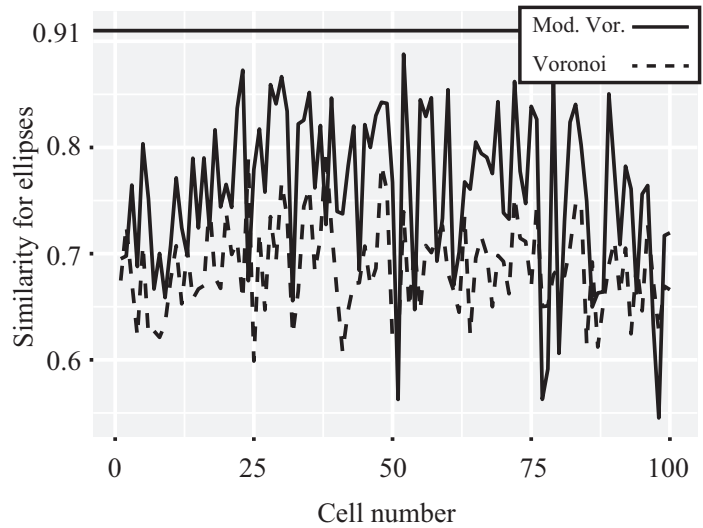

FIGURE 11 Similarity values for the packing of ellipses, with the cases of the original Voronoi diagram (dashed line) and the tessellation obtained by modifying it (continuous line)

For each cell in Figures 4 and 5, the similarity coefficient between the cell and the corresponding ellipse or spherocylinder was calculated. Such calculations were carried out with Monte Carlo methods ${ }^{22}$ for simplicity, and the results can be seen in Figures 11 and 12 for ellipses and spherocylinders, respectively. In these two figures, a similarity coefficient in the modified Voronoi diagram, which is greater than the similarity coefficient of the corresponding Voronoi cell, can be observed in most cases, as expected. The validity of inequalities (3) can also be observed for almost all cells.

The similarity values of the modified Voronoi diagrams are apparently greater than the analogous values in the unmodified Voronoi diagrams, as can be seen in Figures 11, 12, and 15, and the same thing happens between the similarity values of spherocylinders and ellipses in Figure 13. We have chosen the Wilcoxon's signed rank test to confirm these differences, because the distributions of similarity values are skewed. 


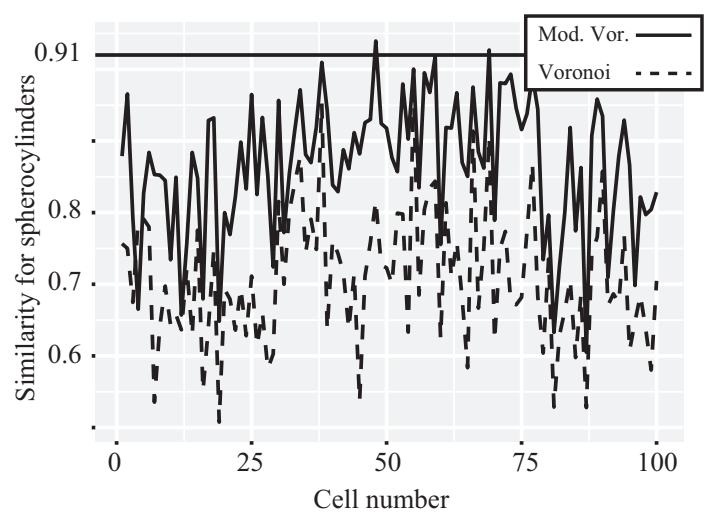

FIGURE 12 Similarity values for the packing of spherocylinders, with the cases of the original Voronoi diagram (dashed line) and the tessellation obtained by modifying it (continuous line)

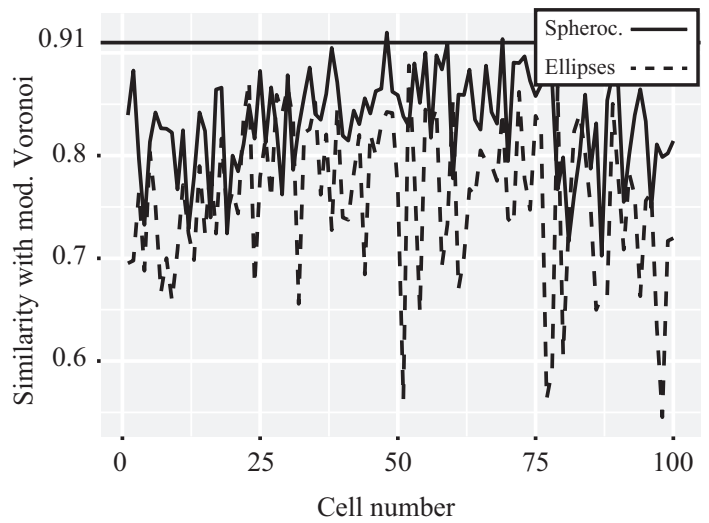

FIGURE 13 Similarity values of the modified Voronoi diagrams in 2D (ellipses and spherocylinders)

According to The Cambridge Dictionary of Statistics, ${ }^{23}$ "the Wilcoxon's signed rank test is a distribution free method for testing the difference between two populations using matched samples. The test is based on the absolute differences of the pairs of observations in the two samples, ranked according to size, with each rank being given the sign of the original difference. The test statistic is the sum of the positive ranks." In the computational implementation of the Wilcoxon's signed rank test used in this paper, ${ }^{24}$ a small p-value corresponding to the test statistic implies a high probability of the populations being different.

The observed difference between the similarity values in the non-Voronoi tessellation and their corresponding Voronoi similarity values is supported by the (paired) Wilcoxon's signed rank test p-values of $3.13 \times 10^{-13}$ and $2.2 \times 10^{-16}$ for the ellipses and spherocylinders, respectively, being the null hypothesis that the distributions of similarity in Voronoi and Laguerre diagrams have the same location, with the alternative hypothesis that the location of the distribution corresponding to Voronoi is smaller than the distribution corresponding to Laguerre.

One more (unpaired) Wilcoxon's signed rank test was also carried out, in order to know if there is any significant difference between the similarity values in the non-Voronoi tessellations (those values can be seen in Figure 13). Such difference can be considered significant, since $8.93 \times 10^{-13}$ is the $\mathrm{p}$-value obtained in the test.

\section{5 | SIMPLE 3D EXAMPLE}

The formulations in the previous section cannot be easily extended to 3D. However, for the sake of completeness, a simple example with spheres is presented (Figure 14). The spheres are contained in a cube and have radii distributed according to the continuous uniform distribution in the interval $[1,2]$. From them, a Voronoi and a Laguerre diagram were obtained with the Voro++ library. ${ }^{7}$ Analogously to the 2D examples, the similarity values (Figure 15) are also statistically different. 


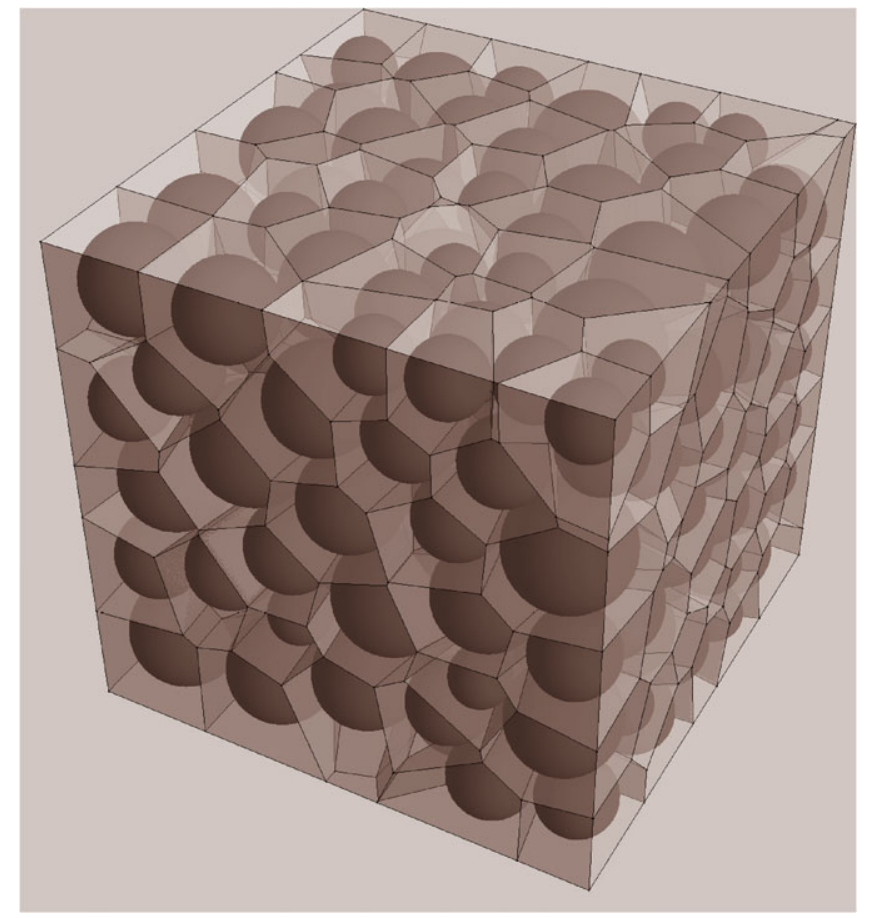

FIGURE 14 Packing of spheres with Laguerre cells superimposed [Colour figure can be viewed at wileyonlinelibrary.com]

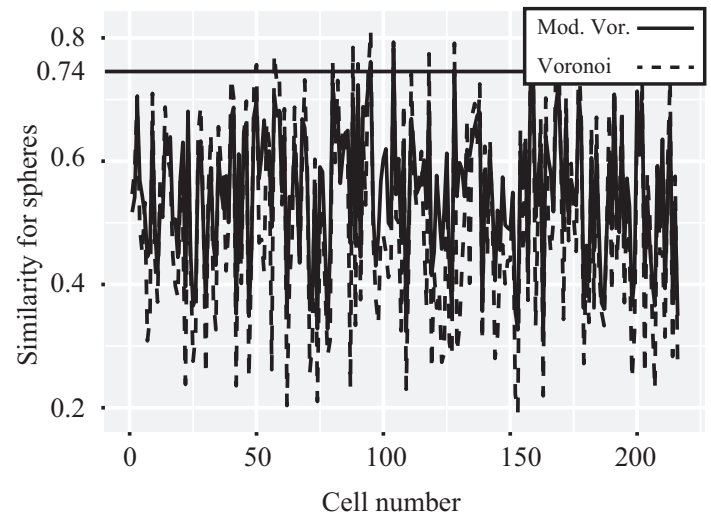

FIGURE 15 Similarity values for the packing of spheres, with the cases of the Voronoi diagram (dashed line) and the power diagram (continuous line)

Despite the similarity values in the last Laguerre diagram can be considered good (notice that here inequalities (3) also hold in most cases), an extension to 3D of the formulations for ellipses and spherocylinders should be carried out in future works. This is because, as previously mentioned, spheres might not be good enough to approximate grain shapes such as the ones in Figure 3. The curves corresponding to the Laguerre and Voronoi similarity values in Figure 15 are not as distinct as the curves in the analogous Figures 11 and 12. This matches the fact that the p-value obtained with the Wilcoxon's test, equal to $2.34 \times 10^{-10}$, is in the order of 1000 times greater than in the other tests.

\section{6 | DISCUSSION}

In this section, we discuss some issues regarding the validity and scope of our approach. It must be said that our results allow to obtain tessellations in order to carry out physical simulations as in the works of Hitti ${ }^{2}$ and Benabbou et al. ${ }^{25} \mathrm{The}$ error or quality of the obtained packings and tessellations can be estimated by measuring properties such as homogeneity, 
isotropy, and space occupation, which should be similar to the real values found in the materials being modeled or simulated. A good source to learn how to make these and other measurements is the work of O'Sullivan. ${ }^{26}$

Our packing algorithm has been validated by comparing it with the results in the work of Benabbou et al, ${ }^{1}$ who also make physical simulations. In this comparison, our packing method yields a higher space occupation for the case of spheres, as can be seen in the work of Valera et al. ${ }^{5}$ However, our best contribution is the generalization of existing results, which allows to obtain tessellations from packings of shapes other than circles or spheres. Further validations would require to carry out physical simulations, as part of a future research.

\section{CONCLUSIONS AND FURTHER WORK}

A modification was proposed to enhance previous algorithms developed by the authors to reproduce the structure of polycrystalline materials in order to better represent packages of elongated particles (grains, crystals, etc). This was achieved by redefining the measurement of distance between the vertices of Laguerre cells and the intermediate bodies (eg, circles) used to represent the areas or volumes of the original particles. In the case of circular bodies, the measure used was the AWPD (length between the cell vertex and the tangential point in the circle) instead of the distance between the vertex and the circle's centers. In the case of noncircular bodies, this concept is further extended in such a way that the particle should be fully encompassed by the surrounding cell. The algorithm was applied to packages of ellipses and spherocylinders in $2 \mathrm{D}$ and to a package of spheres to illustrate a 3D case.

A useful procedure for evaluating the similarity between the cells of a space tessellation and their associated shapes, using the Jaccard coefficient, was presented. In 2D, the similarity values of spherocylinders are higher than the ones for ellipses. This can be seen graphically and analytically. The difference of similarity values in 2D between the Voronoi and the modified Laguerre diagram is greater than in the analogous case of spheres in 3D, but the formulations in 2D, of ellipses and spherocylinders, should be extended anyway to 3D as part of a future work. The Jaccard coefficient used in this paper allows the measurement of tessellations quality as well as choosing better shapes for generating packings used to obtain such tessellations, which are employed for modeling granular materials at the microscale.

The statistic tests randomly carried out on generated packages attest the validity of the proposed methodology. The relative simplicity of the formulations allows them to be adapted to any geometrical shape and is a generalization of previous works with spheres.

\section{ACKNOWLEDGEMENTS}

The authors are deeply grateful to the valuable funding, resources, and support of the following institutions: Coordination for the Improvement of Higher Education Personnel (CAPES), Brazilian Ministry of Education (CAPES-MES Project No. 208/13); InfraLab, Faculty of Technology, University of Brasilia, Brasilia, Brazil; and International Center for Numerical Methods in Engineering (CIMNE), Barcelona, Spain.

\section{ORCID}

Irvin Pérez (iD) https://orcid.org/0000-0001-6211-1468

E. Oñate (D) https://orcid.org/0000-0002-0804-7095

\section{REFERENCES}

1. Benabbou A, Borouchaki H, Laug P, Lu J. Geometrical modeling of granular structures in two and three dimensions. Application to nanostructures. Int J Numer Methods Eng. 2009;80(4):425-454.

2. Hitti MK. Direct Numerical Simulation of Complex Representative Volume Elements (RVEs): Generation, Resolution and Homogenization [PhD thesis]. Paris, France: École Nationale Supérieure des Mines de Paris; 2011.

3. Morfa CR, de Farias MM, Morales IPP, de Navarra EOI, Valera RR. Virtual modeling of polycrystalline structures of materials using particle packing algorithms and Laguerre cells. Comput Part Mech. 2018;5(2):213-226.

4. Blanco PJ, Sánchez PJ, de Souza Neto EA, Feijóo RA. Variational foundations and generalized unified theory of RVE-based multiscale models. Arch Comput Methods Eng. 2016;23(2):191-253.

5. Valera RR, Morales IP, Vanmaercke S, Morfa CR, Cortés LA, Casañas HD-G. Modified algorithm for generating high volume fraction sphere packings. Comput Part Mech. 2015;2(2):161-172. 
6. Rycroft CH. Multiscale Modeling in Granular Flow [PhD thesis]. Cambridge, MA: Department of Mathematics, Massachusetts Institute of Technology; 2007.

7. Rycroft C. Voro++: a three-dimensional Voronoi cell library in C++. http://crd.lbl.gov/departments/applied-mathematics/math/ software/voro-a-three-dimensional-voronoi-library-in-c/. Accessed August 2018.

8. CGAL: the computational geometry algorithms library. https://www.cgal.org/. Accessed August 2018.

9. Qhull. http://www.qhull.org/. Accessed August 2018.

10. Emiris IZ, Tsigaridas EP, Tzoumas GM. Exact Voronoi diagram of smooth convex pseudo-circles: general predicates, and implementation for ellipses. Comput Aided Geom Des. 2013;30(8):760-777.

11. Dong K, Wang C, Yu A. Voronoi analysis of the packings of non-spherical particles. Chem Eng Sci. 2016;153(Suppl C):330-343.

12. Morales IP, de Farias MM, Valera RR, Morfa CR, Martínez Carvajal HE. Contributions to the generalization of advancing front particle packing algorithms. Int J Numer Methods Eng. 2016;107(12):993-1008.

13. Morales IP, Valera RR, Morfa CR, de Farias MM. Dense packing of general-shaped particles using a minimization technique. Comput Part Mech. 2017;4(2):165-179.

14. Deza MM, Deza E. Encyclopedia of Distances. 1st ed. Berlin, Germany: Springer-Verlag Berlin Heidelberg; 2009.

15. Hohenwarter A, Pippan R. Fracture and fracture toughness of nanopolycrystalline metals produced by severe plastic deformation. Philos Trans A Math Phys Eng Sci. 2015;373(2038):20140366.

16. Feng YT, Han K, Owen DRJ. An advancing front packing of polygons, ellipses and spheres. In: Cook BK, Jensen RP, eds. Discrete Element Methods: Numerical Modeling of Discontinua. Santa Fe, NM: American Society of Civil Engineers; 2002:93-98.

17. Levandowsky M, Winter D. Distance between sets. Nature. 1971;234:34-35.

18. Weisstein EW. Circle packing. http://mathworld.wolfram.com/CirclePacking.html. Accessed October 2018.

19. Weisstein EW. Kepler conjecture. http://mathworld.wolfram.com/KeplerConjecture.html. Accessed October 2018.

20. Weisstein EW. Cubic close packing. http://mathworld.wolfram.com/CubicClosePacking.html. Accessed October 2018.

21. Weisstein EW. Hexagonal close packing. http://mathworld.wolfram.com/HexagonalClosePacking.html. Accessed October 2018.

22. Saucier R. Computer generation of statistical distributions. ARL-TR-2168. Army Research Laboratory; 2000. http://disi.unal.edu.co/ gjhernandezp/psc/lectures/04/random.pdf. Accessed October 2018.

23. Everitt BS, Skrondal A. The Cambridge Dictionary of Statistics. 4th ed. Cambridge, UK: Cambridge University Press; 2010.

24. Kabacoff RI. R in Action: Data Analysis and Graphics With R. Shelter Island, NY: Manning Publications Co; 2011.

25. Benabbou A, Borouchaki H, Laug P, Lu J. Numerical modeling of nanostructured materials. Finite Elem Anal Des. 2010;46(1-2):165-180.

26. O'Sullivan C. Particulate Discrete Element Modelling: A Geomechanics Perspective. Abingdon, UK: Spon Press; 2011.

How to cite this article: Pérez I, Muniz de Farias M, Castro M, et al. Modeling polycrystalline materials with elongated grains. Int J Numer Methods Eng. 2018;1-11. https://doi.org/10.1002/nme.6004 